\title{
Quantitative Microstructural Analysis and Modeling of Discontinuously- Reinforced Aluminum (DRA) Composites
}

Jonathan E. Spowart.

UES Incorporated, 4401 Dayton-Xenia Road, Dayton, Ohio 45432-1894.

Microstructural details such as degree of homogeneity and spatial anisotropy of the reinforcement phase can have a profound effect on the mechanical properties of spatially-heterogeneous microstructures such as discontinuously-reinforced aluminum (DRA) and other metallic matrix composites. However, models for predicting the constitutive behavior of based on unit cell approaches generally do not possess the ability to incorporate the effects of these higher-order microstructural features. In addition, currently-available numerical models rarely encompass the volumes of material necessary to ensure statistical relevance. The present work therefore offers an alternative approach for first quantifying and then incorporating microstructural homogeneity into an elastic-plastic finite element (FEA) code, in an attempt to model both the micromechanical length scale associated with the individual reinforcement particles and the microstructural length scale associated with the homogeneity of their spatial distribution, at a greatly-reduced computational expense (Fig. 1). Both 2-D and 3-D models are included to illustrate the flexibility of the technique for dealing with material data sets obtained either via tomography or from serial-sectioning [1].

Using a powder metallurgy approach, DRA composites containing varying levels of microstructural homogeneity were fabricated. In order to quantify the spatial homogeneity of the DRA materials, a modified version of the Multi-Scalar Analysis of Area Fractions (MSAAF) technique originally developed by Spowart et al was used to [2,3]. The modified analysis leads to the definition of two separate (in-plane) homogeneous length scale parameters, $\mathrm{L}_{\mathrm{H} 1}$ and $\mathrm{L}_{\mathrm{H} 2}$, which refer to the spatial homogeneity of the microstructures measured in the axial and transverse directions, respectively.

Automated serial sectioning of the DRA composites was carried out using Robo-Met.3D. Finite element (FEA) models were built from the resulting 2D and 3D data sets, the results of which showed that $\mathrm{L}_{\mathrm{H} 1}$ and $\mathrm{L}_{\mathrm{H} 2}$ have a strong effect on both tensile elastic modulus and yield strength as well as initial strain-hardening rates (Fig. 2). In addition, strain localization and load partitioning were identified as physical mechanisms which strongly contribute to the post-yielding behavior. In addition, when spatial homogeneity is increased there can be a transition from strain-controlled failure mechanisms such as void nucleation and growth towards stress-controlled failure mechanisms such as particle cracking. This provides a clear incentive to produce DRA composites with higher levels of spatial homogeneity, in order to take advantage of other microstructural enhancements such as stronger reinforcement particles and/or stronger interfacial bonding.

This work was supported in part by the Air Force Research Laboratory, Materials \& Manufacturing Directorate, under Air Force Contract Numbers F33615-01-C-5214 and FA8650-04-D-5233.

References

[1] J. E. Spowart, H. M. Mullens, B. T. Puchala, J.O.M., Vol. 55, No. 10, October 2003.

[2] J. E. Spowart, B. Maruyama, D. B. Miracle, Mat. Sci. Eng., Vol. A307, pp. 51-66.

[3] J. E. Spowart, (submitted) Mat. Sci. Engr. (A), (2005) 


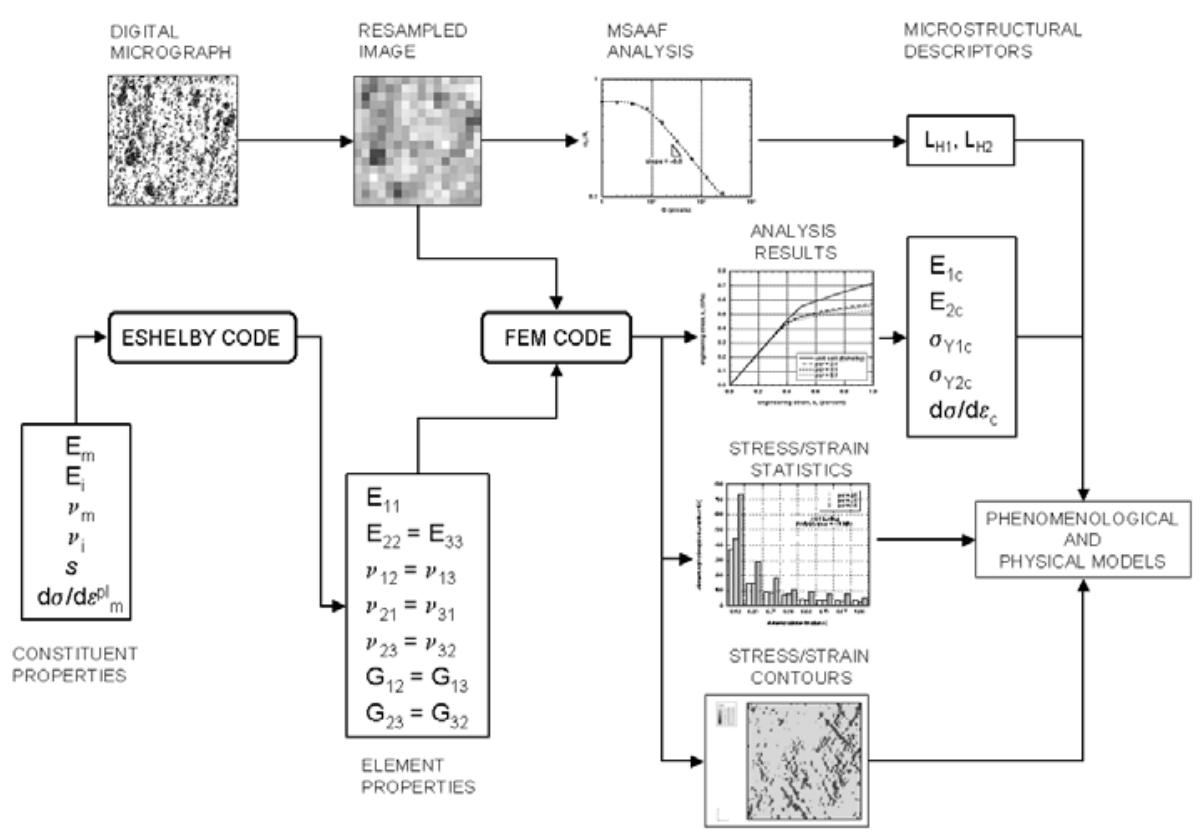

Fig. 1. Details of combined characterization/modeling scheme used to model the DRA composites

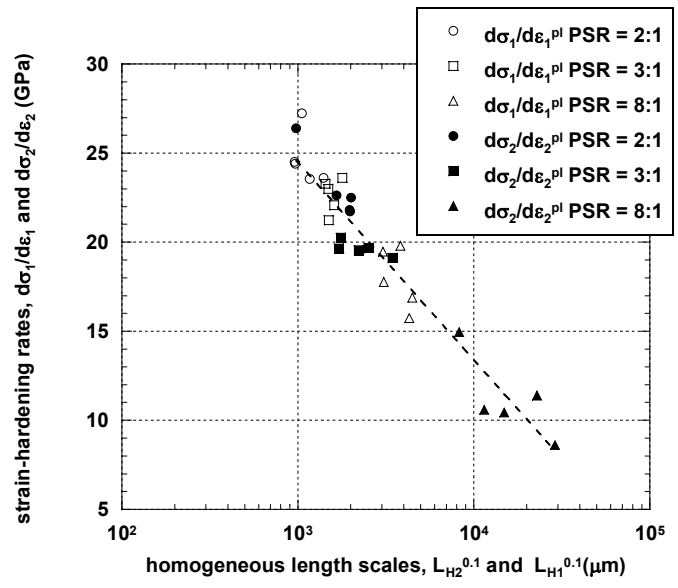

Fig. 2. Relationship between initial strain-hardening rates and homogeneous length scales measured in both axial and transverse directions. The strainhardening data all collapse onto a single line, suggesting that there is a general relationship between the initial strainhardening rate and the spatial homogeneity measured perpendicular to the loading direction.

(a)
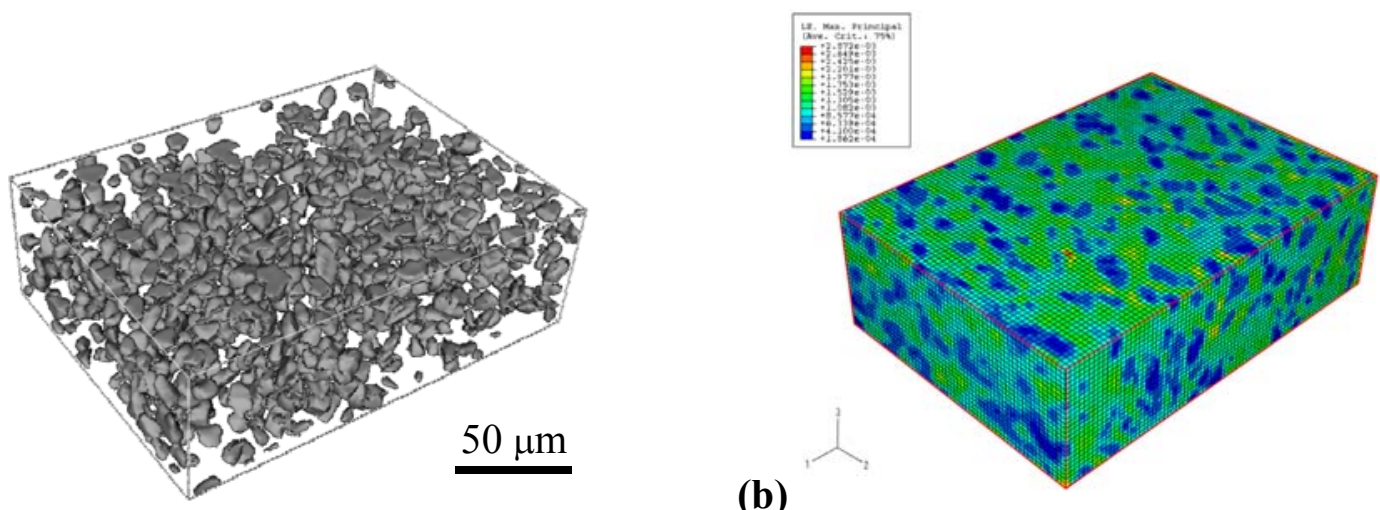

(b)

Fig. 3. 3-D FEA model of DRA microstructure, showing; (a) arrangement of SiC particles, and (b) maximum principal strains after tensile loading parallel to 1 -direction (overall strain $\varepsilon=1 \times 10^{-3}$.) 